\title{
Investigation into Noise Frequency Spectrum Characteristics Corresponding to Blade Nonsynchronous Vibration in Multi-stage Axial Compressor
}

\author{
Yundong Sha ${ }^{1, a}$, Fengtong Zhao', b and Jia Han $^{2}$ \\ ${ }^{1}$ Shenyang Aerospace University, Liaoning Key Laboratory of Digital Technology Simulation \\ and Test Techniques, Liaoning, Shenyang, 110136, P.R. China \\ ${ }^{2}$ Shenyang Aerospace University, School of Aeroengine and Energy Engineering, \\ Liaoning, Shenyang, 110136, P.R. China \\ aydsha2003@vip.sina.com, bftzhao@sina.com
}

\begin{abstract}
Keywords: Multi-stage axial compressor, Nonsynchronous vibration, Noise, Rotating instability, Frequency characteristics
\end{abstract}

\begin{abstract}
Unsteady flow phenomenon occurs in a multi-stage axial compressor. The unsteady flow not only has significant influence on the performance of compressor and the stability of flow, but also can be an excitation source inducing Nonsynchronous vibration (NSV) of rotor blade. NSV is an aeroelastic phenomenon where the rotor blades vibrate at nonintegral multiples of the shaft rotational frequencies in operating regimes where classical flutter is not known to occur. Recently, more and more scholars pay attention to Rotating instabilities (RIs) as one of the unsteady flow phenomenon. RIs have been observed in axial flow fans and centrifugal compressors as well as in low-speed and high-speed axial compressors. They are responsible for the excitation of high amplitude rotor blade vibrations and noise generation. This research aims at revealing the relationships among the unsteady flow behaviors, characteristics of inner sound field and propagation, the vibration of rotor blade in multi-stage axial compressor.

The noise in compressor and the vibration of rotor blade have been measured on a high pressure compressor rig testing. The transducer system is connected to the interior casing wall through the acoustic waveguide pipes. The noise is measured by $1 / 4$ inch condenser microphones in different operating of the compressor. The time-domain wave of noise acquired at different work status of the compressor is transformed into frequency spectrum by Fast Fourier Transform (FFT) to investigate characteristics of sound field in multi-stage axial compressor. And the emphasis is focused on the frequency characteristics of the noise corresponding to blade nonsynchronous vibration.

It is found that the vibration amplitude of the rotor blade suddenly increases in a pre-arranged structure adjustment and specific rotating speed, and noise signal with special frequency structures appears simultaneously. High amplitude levels of blade vibration have occurred on the first rotor of a multi-stage high pressure compressor. The frequencies are not in resonance with harmonics of the rotor speed. The frequency analysis show that the noise has a special frequency structures with combination of the appeared characteristic frequency and the blade pass frequency of rotor blade (BPF). Because the similarities between the frequency combination and the specific frequency structure of the fluctuating pressure when the rotating instability (RI) appears in the axial compressor have been identified, the acting mechanism of rotating instability may exist in this compressor, i.e. the vibrational excitation to the rotor blade may be aerodynamically caused and associated with a rotating flow instability in the compressor.
\end{abstract}

\section{Introduction}

Unsteady flow phenomenon occurs in a multi-stage axial compressor. The unsteady flow not only has significant influence on the performance of compressor and the stability of flow, but also can be an excitation source inducing Nonsynchronous vibration (NSV) of rotor blades. NSV has been observed in an axial compressor rotor blade, which is characterized by being nonsynchronous to the rotor 
speed. i.e., the rotor blades vibrate at nonintegral multiples of the shaft rotational frequencies, and they occur in operating regimes not associated with classical flutter. This type of vibration was previously reported by Baumgartner et al. [1] and Kielb et al. [2]. Recent years, many scholars have paid attention to Rotating instabilities (RIs) as one of the unsteady flow phenomenon. RIs have been observed in axial flow fans and centrifugal compressors as well as in low-speed and high-speed axial compressors. They are responsible for the excitation of high amplitude rotor blade vibrations and noise generation. The relations between tip clearance flow unsteadiness RIs and NSV have been explained with a mechanism based on a novel impinging resonant jet theory by Jean Thomassin et al. [3].

Several researchers have investigated tip clearance flow unsteadiness as the source of noise and NSV, both using computational simulations and experimentation. The representations of these investigations in this area can be found from Kameier and Neise [4,5], Mailach et al. [6], and Liu et al. [7], who studied the tip flow unsteadiness, known as RIs, inferred to excite the casing acoustic modes which in turn can excite the blades. Kameier and Neise investigate the aeroacoustic generation mechanism of the tip clearance noise in a low speed axial turbomachines [8]. They measure the pressure fluctuations on the casing wall close to the impeller and on the rotating blades. The tip clearance noise is found in the acoustic far field only if the rotating blade flow instability components are present simultaneously in the near field. And the characteristic frequency patterns of the pressure fluctuation are found. Baumgartner, Kameier and Hourmouziadis make an experimental investigation about the aerodynamic sources which lead to high non-engine order vibration on the first stage of a multi-stage high pressure compressor [1]. The unsteady pressure is measured at the casing wall just upstream of the first rotor. And pressure fluctuations in the casing wall exhibit a characteristic pattern.

In this paper, the noise in compressor and the vibration of rotor blade have been measured on a high pressure compressor rig testing. NSV of the rotor blade has occurred in a pre-arranged structure adjustment and specific rotating speed, i.e. high amplitude levels of blade vibration have occurred on the first rotor of a multi-stage high pressure compressor. And the frequencies are not in resonance with harmonics of the rotor speed. The frequency analysis show that the noise have a special frequency structures with combination of the appeared characteristic frequencies and the blade pass frequency of rotor blade (BPF).

\section{Experiment Description}

In order to reveal the relationship between rotor blades NSV and the unsteady flow in a multi-stage compressor, investigate the connection between the vibration of rotor blades and the noise signal inner the compressor, an experiment has been made on a multi-stage high pressure compressor rig testing. Sound pressure inner the casing wall, flow field in the vicinity of rotor blades, pressure fluctuations at the casing wall and rotor blade vibration are measured by acoustic waveguide pipes system, Particle Image Velocimetry (PIV), Kulite transducers and vibration measurement system respectively.

This experiment has been conducted according to the acoustic signal measurement technique in the compressor from home and abroad. Sound pressure is measured on four positions of the high pressure compressor, which are selected along the same circumferential direction and the different axial direction position in the compressor. Four positions are respective the clearance of inlet guide vanes (IGV) (position 1), the clearance between inlet guide vanes and the first stage rotor blades (position 2), directly above the first stage rotor blades (position 3 ) and the clearance of the first stage stator blades (position 4). The schematic diagram of measurement positions is showed in Fig.1.

The transducer system is connected to the interior casing wall through the acoustic waveguide pipes before the experiment. The waveguide pipes are mounted flush with the interior casing wall. And the noise is measured by $1 / 4$ inch condenser microphones. The block diagram of the measurement system is showed in Fig.2. 


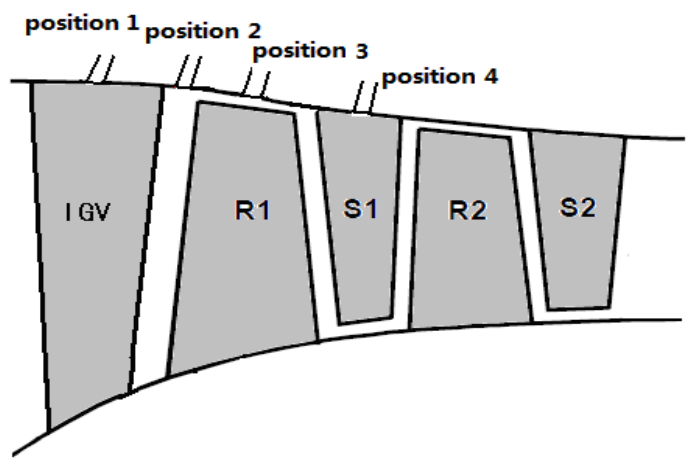

Fig. 1 Schematic diagram of measurement positions

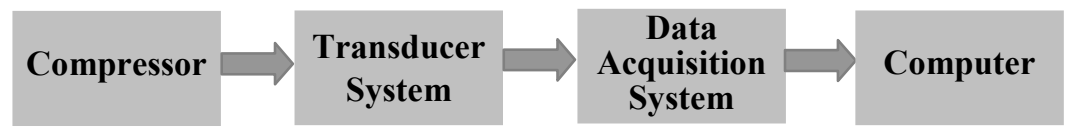

Fig. 2 Block diagram of measurement system

In order to improve the measurement precision, the measurement system is calibrated before the experiment. Considering the need and the possible error of the experiment, the sampling frequency is set to $25.6 \mathrm{kHz}$, which can ensure the acquisition of high frequency sound signal.

In this experiment, several structure adjustments are achieved through adjusting deflecting angles of (IGV) and stator adjustable vanes. And main performance parameters of the compressor are regulated, which include rotating speed $(\mathrm{N})$, corrected mass flow rate $(\alpha)$, increasing pressure ratio $\left(\pi_{c}\right)$ and efficiency $(\eta)$. Changeable characteristics of noise signal inner the compressor are monitored in diverse pre-arranged structure adjustments. And the emphasis is focused on the frequency characteristics of the noise corresponding to blade nonsynchronous vibration.

\section{Data processing and Analysis}

Sound pressure data are measured in diverse pre-arranged structure adjustment and rotating speed through adjusting main performance parameters and deflecting angles of (IGV) and stator adjustable vanes. It is found that high amplitude vibration of rotor blades occurs when the deflecting angle of (IGV) is $0^{\circ}, \alpha_{2}$ is original state 26 and rotating speed of high pressure rotors is near to $9930 \mathrm{r} / \mathrm{min}$.

And noise signal with special frequency structures appears simultaneously. Time-domain waves of special frequency noise signal in four measured positions are shown in Fig.3 under high amplitude vibration of rotor blades.

Frequency Spectrum Analysis of the Noise Corresponding to Rotor Blades NSV. It is found that the vibration amplitude of rotor blade suddenly increases in a pre-arranged structure adjustment and specific rotating speed, and noise signal with special frequency structures appears simultaneously. High amplitude levels of blade vibration have occurred on the first rotor of a multi-stage high pressure compressor. The frequencies are not in resonance with harmonics of the rotor speed. This vibration is just NSV. In order to reveal the special frequency structures under high amplitude vibration of rotor blades, the time-domain wave of noise is transformed into frequency spectrum by FFT. The frequency interval of spectrum analysis is $25 \mathrm{~Hz}$. Frequency spectra of characteristic frequency noise signal are shown in Fig.4 under high amplitude vibration of rotor blades.

It is analyzed that characteristic frequency $1402 \mathrm{~Hz}$ has appeared in every noise signal of measured position when high amplitude vibration of rotor blades occurs in the above adjustable status. (1352 $\mathrm{Hz}$ appears in position 1). In Fig.4, characteristic frequency $4883 \mathrm{~Hz}$ has appeared in the other three positions except position 2, $6285 \mathrm{~Hz}$ is the blades passing frequency of rotor blades (1BPF), and $12570 \mathrm{~Hz}$ is $2 \mathrm{BPF}$. It is found that specific combinations exist between these characteristic frequencies and BPF, i.e. $6285 \mathrm{~Hz}-1402 \mathrm{~Hz}=4883 \mathrm{~Hz}, 6285 \mathrm{~Hz}+1402 \mathrm{~Hz}=7687 \mathrm{~Hz}(7712 \mathrm{~Hz})$. 


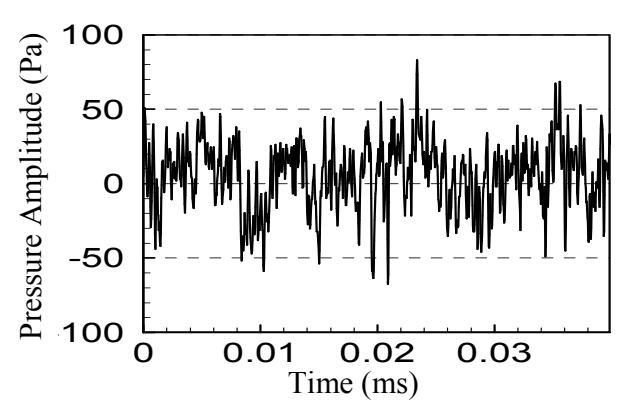

(a)

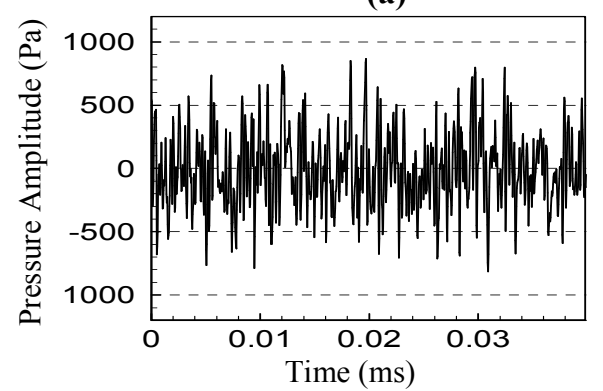

(c)

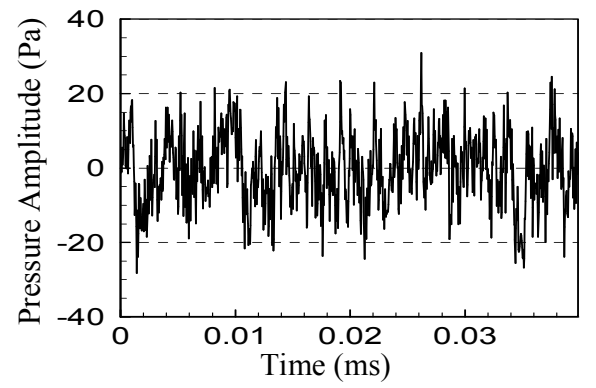

(b)

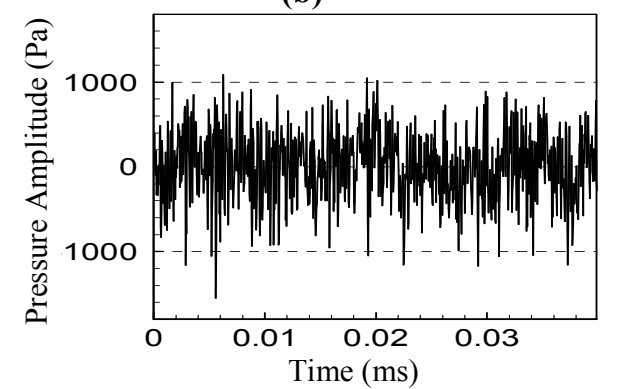

(d)

Fig. 3 Noise in four positions under high amplitude vibration of rotor blades

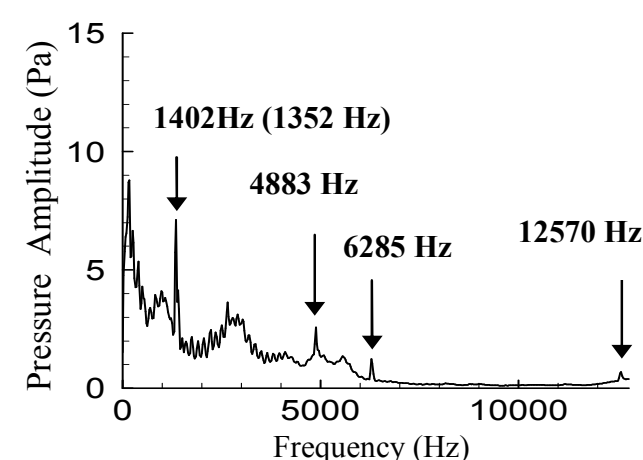

(a)

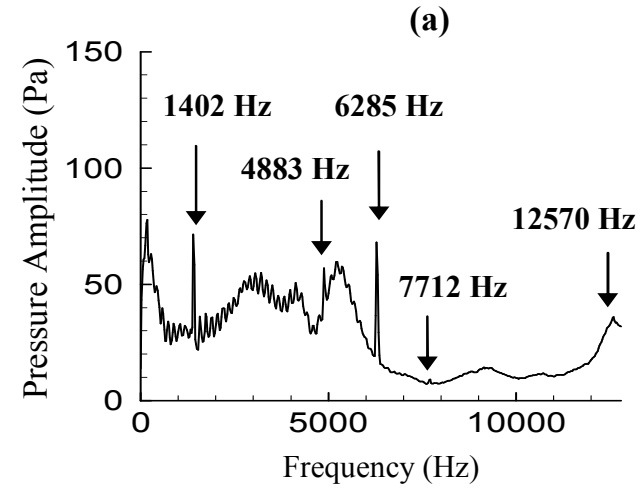

(c)

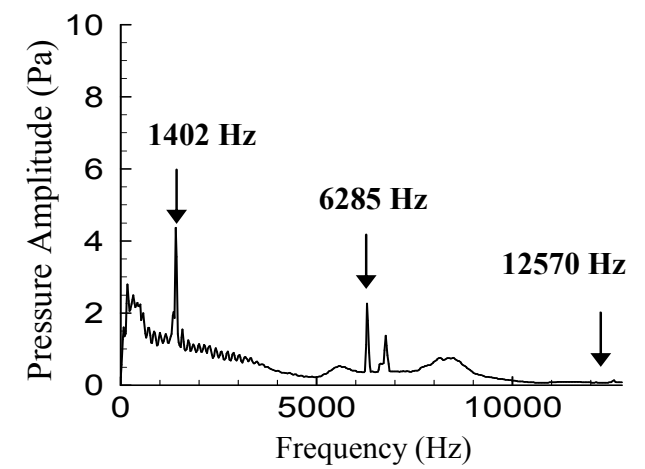

(b)

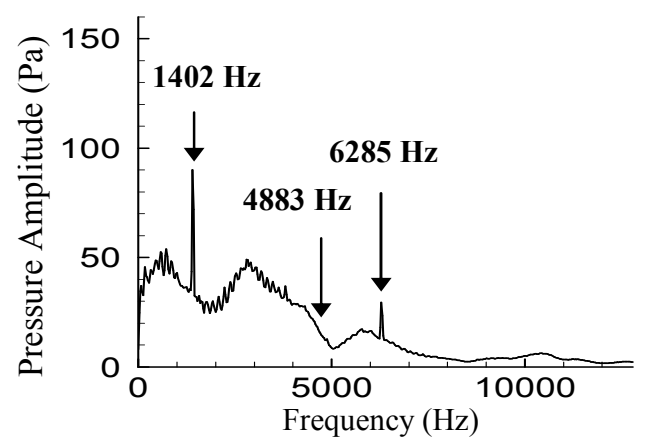

(d)

Fig. 4 Frequency spectra of characteristic frequency noise in four positions under high amplitude vibration of rotor blades

Sound Pressure Level Analysis at 1402 Hz. $1402 \mathrm{~Hz}$ occurs in characteristic frequency spectrum in a pre-arranged structure adjustment and specific rotating speed. Several different rotating speed statuses have been monitored to reveal the sound pressure level at $1402 \mathrm{~Hz}$ (Table. 1 and Fig.5).

In Table. 1 and Fig.5, it can be found that SPL at $1402 \mathrm{~Hz}$ presents first increases then decrease accompanying with the increase of rotating speed. And SPL at $1402 \mathrm{~Hz}$ reaches the higher values at the rotating speed between $9780 \mathrm{r} / \mathrm{min}-9960 \mathrm{r} / \mathrm{min}$. The highest SPL is $128 \mathrm{~dB}$. 
Table. $1 \mathrm{SPL}$ at $1402 \mathrm{~Hz}$ at different rotating speed

\begin{tabular}{cccc}
\hline $\begin{array}{c}\mathrm{N}_{2} \\
(\mathbf{r} / \mathbf{m i n})\end{array}$ & $\begin{array}{c}\mathbf{S P L} \\
(\mathbf{d B})\end{array}$ & $\begin{array}{c}\mathrm{N}_{2} \\
(\mathbf{r} / \mathbf{m i n})\end{array}$ & $\begin{array}{c}\mathbf{S P L} \\
(\mathbf{d B})\end{array}$ \\
\hline 9480 & 119.44 & 10080 & 116.97 \\
9540 & 119.07 & 10140 & 116.01 \\
9600 & 119.36 & 10200 & 116.28 \\
9660 & 119.01 & 10260 & 115.82 \\
9720 & 119.74 & 10320 & 115.39 \\
9780 & 125.24 & 10380 & 115.51 \\
9840 & 126.03 & 10440 & 114.74 \\
9900 & 127.69 & 10500 & 113.88 \\
9960 & 126.89 & 10560 & 111.67 \\
10020 & 119.51 & - & - \\
\hline
\end{tabular}

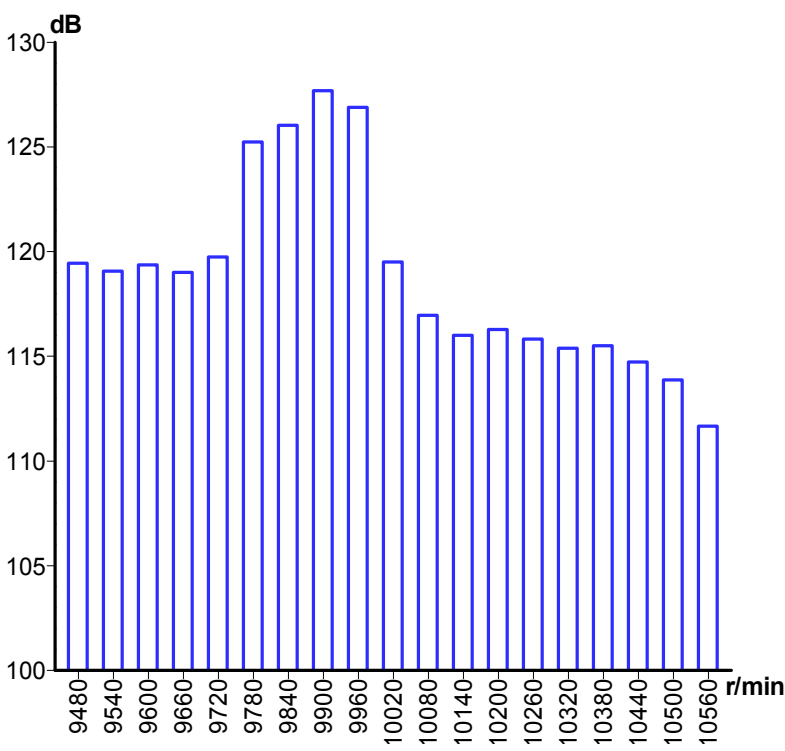

Fig. 5 SPL at $1402 \mathrm{~Hz}$ at different rotating speed

Comparison with Typical Pressure Spectra at the Casing Wall. It is mentioned in the introduction that Kameier and Neise investigate the aeroacoustic generation mechanism of the tip clearance noise in a low speed axial turbomachines. They measure the pressure fluctuations on the casing wall close to the impeller and on the rotating blades. The generation of tip clearance noise is explained by the interaction of rotating instability component with the impeller blades in their paper. And the specific frequency patterns of the pressure fluctuation are found (Fig.6 left diagram). They define the special frequency as characteristic frequency of RI. Baumgartner et at. make an experimental investigation about the aerodynamic sources which lead to high non-engine order vibration on the first stage of a multi-stage high pressure compressor. The unsteady pressure is measured at the casing wall just upstream of the first rotor. And pressure fluctuations in the casing wall exhibit a characteristic pattern (Fig.6 right diagram). This characteristic frequency of RI is thought of the vibrational excitation of high amplitude levels of blade vibration in their research.

The vibration amplitude of the rotor blade suddenly increases in a pre-arranged structure adjustment and specific rotating speed, and noise signal with special frequency structures occurs in the compressor (Fig.7).
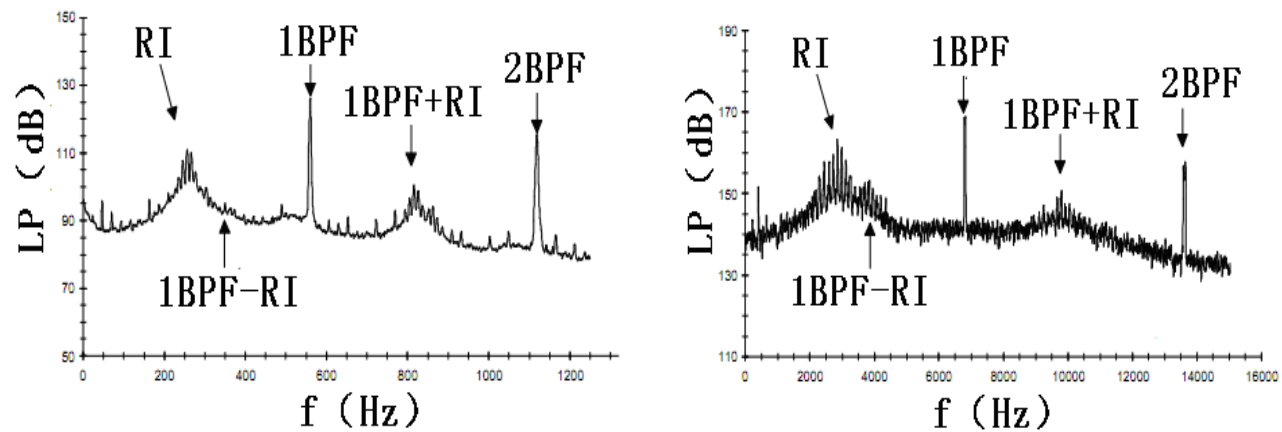

Fig. 6 Pressure spectra at the casing wall, upstream the leading edge of the rotor; left diagram: low- speed compressor; right diagram: first stage of a multi-stage high-speed compressor

In Fig.7, it is shown that $1402 \mathrm{~Hz}$ is defined as Special Frequency (SF). The results tie up very well with Fig.6 on a low speed and on a multi-stage high pressure compressor. And SF is just similitude as RI. So the acting mechanism of rotating instability may exist in this compressor, i.e. the vibrational excitation to the rotor blade may be aerodynamically caused and associated with a rotating flow instability in the compressor. 


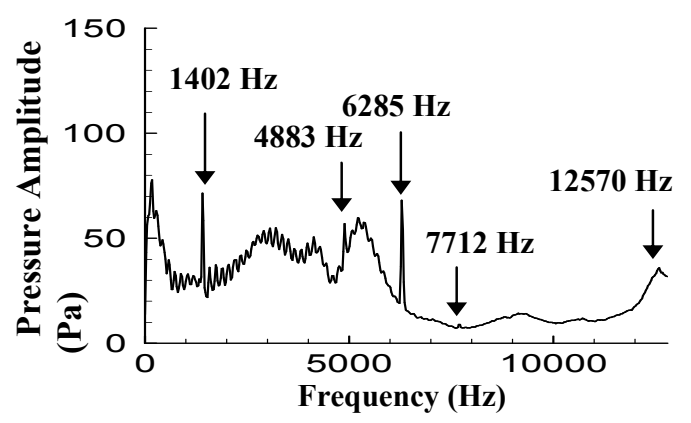

Fig. 7 Noise pressure spectra inner the casing wall, top of the first stage rotor in a multi-stage compressor of the present investigation

\section{Conclusions}

An experimental investigation on a multi-stage compressor shows that for certain operating condition high level vibration on the first stage rotor blades exist. The amplitudes are not in resonance with engine orders. Sound pressure fluctuations in the casing wall exhibit a characteristic pattern.

The vibration amplitude of the rotor blade suddenly increases and noise signal with special frequency structures appears simultaneously. The noise has a special frequency structures with combination of the appeared characteristic frequencies and the blade pass frequency of rotor blade (BPF). SPL at $1402 \mathrm{~Hz}$ presents first increases then reduce accompanying with the increase of rotating speed, which reaches the highest value $128 \mathrm{~dB}$ at $9960 \mathrm{r} / \mathrm{min}$. The acting mechanism of frequency connections will be investigated thoroughly.

By comparing the specific noise spectra with the typical frequency spectra for RI when rotor blades NSV occur, the conclusion can be drived that the acting mechanism of rotating instability may exist in this compressor, i.e. the vibrational excitation to the rotor blade may be aerodynamically caused and associated with a rotating flow instability in the compressor.

\section{References}

[1] M. Baumgartner, F. Kameier and J. Hourmouziadis, Non-Engine Order Blade Vibration in a High Pressure Compressor, 12th International Symposium on Airbreathing Engines, 1995, pp. $10-15$.

[2] R.E. Kielb, J.P. Thomas, J.W. Barter and K.C. Hall, Blade Excitation by Aerodynamic Instabilities-A Compressor Blade Study, Journal of Turbomachinery Paper No. GT-2003-38634.

[3] Jean Thomassin, Huu Duc Vo, and Njuki W. Mureithi, Blade Tip Clearance Flow and Compressor Nonsynchronous Vibrations: The Jet Core Feedback Theory as the Coupling Mechanism, Journal of Turbomachinery, 2009, Vol.131/011013-1.

[4] F. Kameier, and W. Neise, Rotating Blade Flow Instability as a Source of Noise in Axial Turbomachines, Journal of Sound and Vibration, 1997, pp. 833-853.

[5] F. Kameier, and W. Neise, Experimental Study of Tip Clearance Losses and Noise in Axial Turbomachines and Their Reduction, Journal of Turbomachinery. 1997, 119, pp. 460-471.

[6] R. Mailach, I. Lehman and K. Vogeler, Rotating Instabilities in a Axial Compressor Originating From the Blade Tip Vortex, Journal of Turbomachinery, 2001, 123, pp. 453-463.

[7] J.M. Liu, F. Holste and W. Neise, On the Azimuthal Mode Structure of Rotating Blade Flow Instabilities in Axial Turbomachines, AIAA and CEAS Aeroacoustics Conference, 1996, pp. 96-1741.

[8] F. Kameier, and W. Neise, Reduction of Tip Clearance Losses and Noise in Axial-Flow Machines, AGARD PEP 85th Meeting on Loss Mechanisms and Unsteady Flows in Turbomachines, 1995, pp. 8-12. 